\title{
Erratum a: Valutazione di un nuovo metodo per la tipizzazione HLA nella malattia celiaca
}

Lucia Terzuoli • Brunetta Porcelli •

Maria Teresa Trevisan · Alicia Benedetti · Elio Tonutti

Pubblicato online: 22 ottobre 2013

(C) Springer-Verlag Italia 2013

Erratum a: Riv Ital Med Lab (2013) 9:149-154

DOI 10.1007/s13631-013-0016-0

Nella versione originale di questo articolo è contenuto un refuso accidentale: il nome della quarta autrice è Alice Benedetti e non Alicia Benedetti.

Ci scusiamo con i Lettori.

La versione online dell' articolo originale può essere trovata doi:10.1007/s13631-013-0016-0.

L. Terzuoli $(\varangle) \cdot$ B. Porcelli

Dipartimento di Biotecnologie Mediche, Università degli Studi di

Siena, Via A. Moro 2, 53100 Siena, Italia

e-mail: lucia.terzuoli@unisi.it

L. Terzuoli · B. Porcelli

UOC Patologia Clinica, Azienda Ospedaliera Universitaria

Senese, Siena, Italia

M.T. Trevisan · A. Benedetti

Laboratorio Analisi, Ospedale G. Fracastoro, San Bonifacio, VR,

ULSS 20, Verona, Italia

E. Tonutti

Laboratorio di Immunopatologia e Allergologia, Azienda

Ospedaliero-Universitaria, Udine, Italia 